\title{
X-ray Detectors - in Heaven and on Earth
}

\author{
Lothar Strüder ${ }^{1,3}$, Heike Soltau ${ }^{2}$, Peter Holl ${ }^{1}$, Robert Hartmann ${ }^{1}$, Dieter Schlosser ${ }^{1}$ Adrian Niculae ${ }^{2}$, \\ Martin Huth ${ }^{2}$ and Jeff Davis ${ }^{2}$ \\ 1. PNSensor GmbH, Otto-Hahn-Ring 6, Munich, Germany \\ 2. PNDetector GmbH, Otto-Hahn-Ring 6, Munich, Germany \\ 3. University of Siegen, Physics Department, Siegen, Germany
}

While the astrophysical objects are observed on a length scale of $10^{24} \mathrm{~m}$ to $10^{9} \mathrm{~m}$ the X-ray measurements on Earth range from $10^{0} \mathrm{~m}$ down to $10^{-12} \mathrm{~m}$. Although the length scale is very different the techniques used show a lot of commonalities. The precise knowledge of the distribution, the density, structure and the chemical composition of matter is not only of major importance in many scientific fields, but equally in industrial applications. The analysis of X-rays in astronomy performed in space - is used to derive for example composition and temperature of elements in supernova remnants, velocities of celestial sources, ionization states, ionization mechanisms, relaxation processes and atomic processes in hot plasmas. X-ray fluorescence or X-ray diffraction from matter on Earth is used to study its composition, structure and dynamics. In the fields ranging from physics and chemistry to material and life sciences X-ray techniques are able to deliver meaningful answers to fundamental questions. In addition, quality assurance and control in industrial processes are very often performed with methods making use of X-ray fluorescence and diffraction.

The generation mechanisms of the exciting radiation are quite different in both fields: In astrophysics the X-rays can be generated through high temperatures, rapidly spinning objects, collision of matter, etc. while on Earth dedicated controlled instruments like X-ray tubes, synchrotrons, laser plasma or accelerator driven sources and charged particles (PIXE) are used. In both cases the experimental techniques for the detection of the X-rays are similar while the scientific questions are very different. While in X-ray astronomy gigantic X-ray mirrors are used to focus the incoming light onto the focal plane on Earth KB-mirrors, zone plates, crystals and gratings are used to guide the X-rays to the detector. Despite of the large mirrors in astronomy only a few X-rays are reaching the detector per second while it can be as high as $10^{9}$ X-rays in the case of terrestrial applications. The desired common features of the detectors for both areas "Heaven and Earth" are: (1) high quantum efficiency, (2) low electronic noise at high readout speed translating in good energy resolution, (3) adjusted spatial resolution, (4) large areas with high fill factor, (5) good long-term stability, high radiation hardness, (6) low detector generated background and (7) high dynamic range. Although the ranking of (1) to (7) is different in the various fields of applications they all play a key role during the development of the detectors. Four types of silicon X-ray radiation detectors will be presented including their performance in X-ray satellite missions and measurements in solid state and atomic physics, material science and the study of soft matter: PIN and PAD detectors (1), Silicon Drift detectors (2), Charge Coupled Devices (3), hybrid pixel detectors and active pixel sensors (4).

PIN and PAD detectors were first used for X-ray measurements in space on a Mars mission for the analysis of the Martian soil, at a later stage replaced by Silicon Drift Detectors. On Earth the PIN detectors are still used as a cheap X-ray detector in hand-held instruments with limited performance in speed and energy resolution. Silicon drift detectors [1] have replaced almost completely the $\mathrm{Si}(\mathrm{Li})$ detector in XRF and microanalysis measurements. They exhibit Fano limited energy resolution in a 
wide energy range and high count rate capability without the need of cryogenic cooling. CCDs have been first used on large X-ray missions aboard Chandra and XMM-Newton [2] where they delivered spectacular spectroscopic images since the year 2000. On Earth similar fully depleted backilluminated pnCCDs are used at Free Electron Laser facilities, synchrotrons and in many laboratory set-ups for spectroscopy and imaging [3]. pnCCDs for example are equally used as direct electron detectors in transmission electron microscopes. Hybrid pixel detectors and active pixel sensors are not yet in space on major satellites but in preparation for future X-ray missions. On ground they are employed at synchrotrons, free electron lasers [4] and in electron microscopy. For all of those wide spread uses of the different X-ray detectors examples will be shown and the different optimizations and trade-offs for their specific use will be highlighted.

Fig.1 shows an X-ray image from the supernova remnant [4], first observed by Tycho Brahe in 1572 in the visible domain. A variety of features can be seen: The Bremsstrahlung and thermal radiation from the object, as well as the abundances of fluorescence lines of $\mathrm{O}, \mathrm{Ne}, \mathrm{Mg}, \mathrm{Si}, \mathrm{S}, \mathrm{Ca}$, and Fe. As the distribution of the fluorescence lines are spatially resolved the evolution and the dynamics of the explosion can be understood. Fig. 2 shows a diffraction pattern of a virus, recorded at the Linac Coherent Light source at SLAC [5]. The reconstructed images from 200.000 shots was delivering a spatially resolved 3- dimensional representation of the virus with $20 \mathrm{~nm}$ resolution.

References:

[1] A. Niculae et al., Microscopy and Microanalysis 22 (S3) (2016), p. 40.

[2] L. Strüder et al., Rev. Sci. Instrum. 68 (11), (1997)

[3] G. Lutz et al., Nuclear Inst. and Methods in Physics Research, A, Volume 845 (2017), p. 122.

[4] A Decourchelle et al., A\&A 365 (2001), p. L218.

[5] M Seibert et al., Nature, 470 (7332) (2011), p. 78.
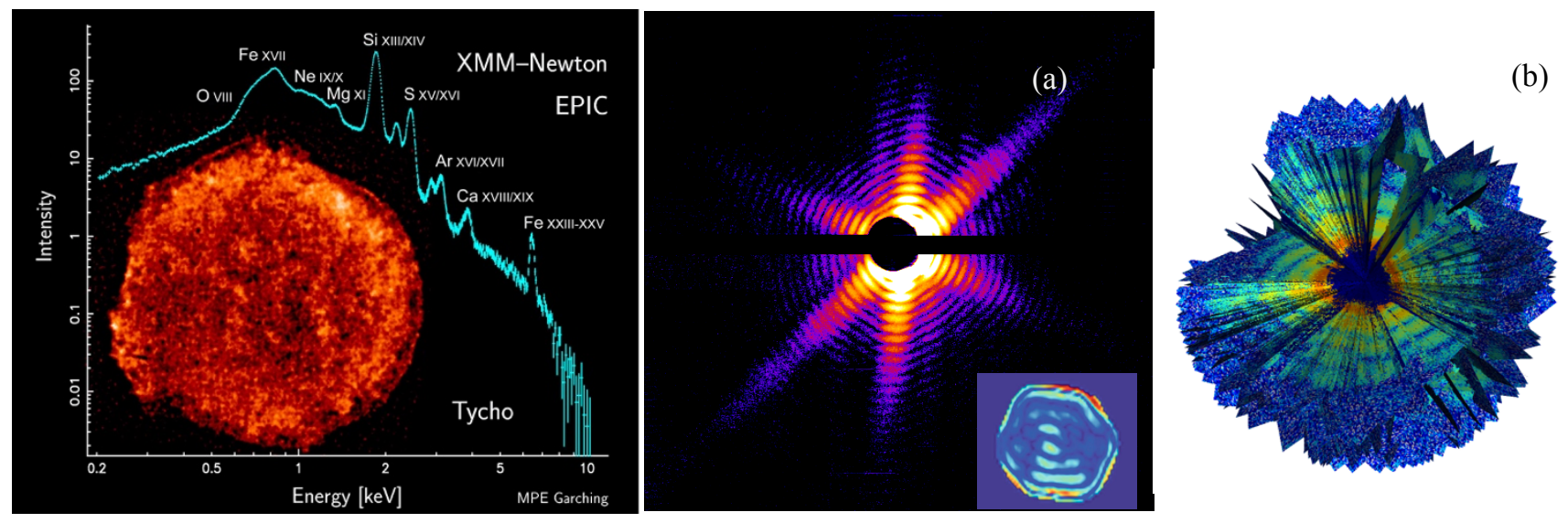

Figure 1. X-ray emission from the supernova Tycho Brahe recorded with the X-ray satellite XMM-Newton
Figure 2 (a) and (b). (a) Diffraction pattern of a virus recorded at LCLS at an X-ray energy of $1.8 \mathrm{keV}$. The spatial resolution is $20 \mathrm{~nm}$. The insert shows the 2-dim. reconstructed image, (b) a 3-dim. representation 